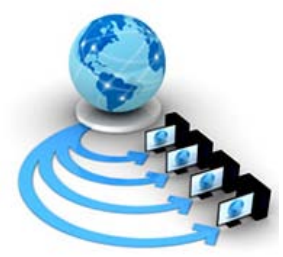

International Journal of Advanced Research in Computer Science

RESEARCH PAPER

\title{
AN IMPROVED TRUST MODEL FOR MITIGATING DISHONEST RECOMMENDATION PROBLEM IN MANET
}

\author{
Kefayat Ullah \\ Department of Computer Science \\ Assam University, Silchar-788011, India
}

\author{
Prodipto Das \\ Department of Computer Science \\ Assam University, Silchar-788011, India
}

\begin{abstract}
MANET is a wireless network with mobile nodes without having any prior infrastructure setup. MANET does not require any access point, router, server etc. The nodes of the MANET communicate themselves through intermediate hop nodes. Each node in MANET acts both as host and also router. This is a self-organized and self-configured network. They use wireless channels randomly for communication. MANETs have many different features as compared to infrastructure network like dynamic topology, nodes higher mobility, low bandwidth, low battery power, higher error rates etc. The nodes in MANET can be connected anywhere any time arbitrarily due to its dynamic features. Also nodes in MANET act as a router and participate in route discovery and maintenance. Security is a major issue in MANET. MANET is vulnerable to different types of attack due to its features like dynamic topology, limited bandwidth, battery power etc. The attackers can attack in various ways, like sending fake routing information, fake messages, flooding packets, flooding false packets etc. There are various types of attack in each layer of the network. Different trust models have been developed for securing the network using recommendations from other nodes, but dishonest recommendations may lead to take wrong decision. This paper presents a probability based trust model which uses recommendations from other nodes. Here, the possible attacks that can occur due to dishonest nodes while taking recommendation are investigated. Here, a filtering algorithm is proposed for resisting the effect of misbehaving nodes while taking recommendations for selecting valid nodes for recommendation.
\end{abstract}

Keywords: MANET, Trust, Recommendation, reputation, DSR.

\section{INTRODUCTION}

Many researchers have given the definition of trusts [1] in different terms such as risk, belief, probability, quality of services etc. Also the trust can be achieved through different ways such as using reputation function, calculating direct trust, calculating trust through recommendation. Many researchers have used recommendation based trust model for filtering the malicious nodes. Li et al. [2] proposed recommendation based trust model to screen the misbehaving nodes. But while taking recommendation from other nodes, it is difficult to filter out the malicious nodes from recommendation and dishonest recommendation may yield wrong trust. Dishonest recommendation may cause various types of attacks like collusion, bad mounting and ballot stuffing which may cause malfunctioning of trust frameworks. Some researchers have given various solutions for filtering the misbehaving nodes while recommending the trustor nodes but these are very limited and not much effective. Yu et al. [3] proposes one approach to judge the goodness of recommending nodes by taking majority opinion from the recommenders. In this case screening out the malicious nodes is difficult when dishonest recommenders collude each other to perform a malicious attack. Zouridaki et al. [4] proposes service reputation approach which uses recommendations from nodes which have higher trust values. But a node can be more trusted in terms of packet forwarding while may not be a trusted node for recommendation. Buchegger and Boudee [5] proposed a trust model based on experiences to screen out the malicious nodes which are not compatible to evaluating nodes. This approach may not work when the trustee node has no prior experiences to the trust evaluating node. Thus resulting a confusing trust model for evaluating nodes trustiness.
In this paper a recommendation based trust model is presented which excludes dishonest recommendation for evaluating trust. Here first we consider the majority rule to assure the consistency of recommendations in terms of time and location, personal experience based rule to assure the consistency of recommendation with experience held by the evaluating nodes and the service reputation based rule to assure the honesty of the recommender nodes. Second, a defense scheme is proposed to estimate the trustiness of the recommender using the parameters number of interactions, compatibility to the evaluating node using deviation test and association between nodes. The defense scheme considers social properties for estimating trustiness. For computing the performance of the algorithm different mobile nodes are taken into consideration against different mobile topologies.

\section{DIFFERENT TYPE OF ATTACKS RELATED TO RECOMMENDATION IN TRUST FRAMEWORKS}

In present days it's a great challenge to safeguard a network against various attacks. Recent researches are going on to tackle the dishonest nodes in terms of packet forwarding such as blackhole[6], wormhole[7], grayhole[8] etc. It is also of great importance that trusts management frameworks are prone to attack by means of dishonest recommendations. There are various attacks at the time of recommendation propagation and aggregation such as bad mouthing attack, intelligent behaviour attack, ballot stuffing attack, selective misbehaviour attack, time-dependent attack and locationdependent attack. The attacks are explained below:

a) Bad Mouthing Attack (BMA): In BMA the colluding nodes gives negative ratings to good nodes in order to fade the reputation of the nodes. Such malicious behaviour confused the trust management framework. 
b) Ballot Stuffing Attack (BSA): In BSA the collusive nodes mislead the trust mechanism by propagating fake positive rating to some low performing nodes.

c) Selective Misbehaviour Attack (SMA): This attack illtreats some selective good nodes by false rating, but act normally to other nodes. These types of nodes are very difficult to detect for trust mechanism.

d) Intelligent Behaviour Attack (IBA): IBA gives high or low rating recommendation as per threshold. Such type of attack can perform malicious activity by dynamically responding to the threshold values.

e) Time-dependent Attack (TDA): In this type of attack nodes behave correctly for a certain period of time but change their behavior at other times.

f) Location-dependent Attack (LDA): In this type of attack nodes act differently at different location which affect the mobility property of MANET.

Hoffman et al. [9] give a solution to such type of attack by using Bayesian statistical theory for computing the correctness of the recommendations. Some researchers have used majority opinion technique, fixing a threshold for positive and negative recommendation, sufficient interaction history etc. to correctly evaluate the honesty of the recommendation nodes but not sufficient till today. From the literature study we can say that trustiness of recommending nodes cannot be measured by a single approach, it should be done by using multiple properties like time, location, closeness between nodes, which is not present in the literature. With the purpose to improve the correctness and robustness of trust model, the effect of untrusted recommendation should be avoided.

\section{THE PROPOSED TRUST MODEL}

This section presents the proposed trust model which uses both direct and recommendation based trust value of each node to secure the MANET routing protocol. The proposed model has taken into consideration about the attacks discussed above occurs due to some dishonest nodes. The model used the same trust evaluating function for evaluating the trust value of each node, where we have considered both direct and indirect trust calculation function. The model addressed two types of attacks bad mounting and ballot stuffing to assess the functionality of the model. The bad mouthing and ballot stuffing are two types of attacks which occurred due to dishonest recommendation problem. The proposed model uses two components namely Trust Computation Module and Recommendation Manager Model.

\subsection{A. Trust Computation Module}

The Trust Computation Module uses direct as well as indirect trust for calculating the trust as explained below. The trust model uses direct trust when there is a pre initiated trust relationship between the trustor and trustee nodes. The trust computation module uses indirect trust when there is no direct trust value that there is no previous trust relationship exists. In such cases taking reference is important for calculating the trustiness of the nodes, but taking recommendation from all nodes may leads to malicious attacks. The attacker nodes may intentionally propagate dishonest recommendation for referring wrong route. The final trust value is calculated by combining both the direct and indirect trust. $\mathrm{X}_{\mathrm{ij}}$ is positive experiences and $Y_{i j}$ is the negative experience of $i$ on $j$.

The direct trust can be calculated using the following formula

$$
D T_{i j}=\frac{X_{i j}}{X_{i j}+Y_{i j}} \quad \text { Where, } \quad 0 \leq D T \leq 1
$$

The recommendation trust can be calculated using the formula

$$
R T_{i j}=\frac{\sum_{k=1}^{n} X_{k j}}{\sum_{k=1}^{n}\left(X_{k j}+Y_{k j}\right)} \quad \text { Where, } \quad 0 \leq R T \leq 1
$$

Indirect trust is calculated based on the sum of received recommendation in the form of ratings $\left(X_{k j}, Y_{k j}\right)$.

The Final Trust will be calculated as

$$
\begin{array}{r}
F T_{i j}=W_{d} * D T_{i j}+W_{i} * R T_{r} \text { Where, } 0 \leq D T \leq 1 \\
, 0 \leq R T \leq 1, \text { and } D T+R T=1(3)
\end{array}
$$

Where $\mathrm{W}_{\mathrm{d}}$ is the weight of the direct trust and $\mathrm{W}_{\mathrm{i}}$ is the weight of the indirect trust.

\subsection{B. Recommendation Manager Module}

The recommendation manager module sends recommendation request and collect recommendations for a node from a list of recommender nodes. It is designed mainly to detect and exclude the false recommendations. The recommendation manager module first sends recommendation request to the evaluating node's neighbours; gathered received recommendations from the neighbours and runs the filtering algorithm. After running the filtering algorithm it sent back to the trust computation module a list of honest recommendations. Finally using the honest recommendations the evaluating node compute the trust value of a particular node.

Algorithm 1: Recommendation Manager Algorithm

Step 1: For each RecRequest from $\mathrm{S}$ the Recommendation Manager broadcasts

$$
\{
$$

Step 2: RecRequest $\rightarrow$ neighbours

Recommendation Request

Step 3: Gather received recommendation

Step 4: Construct RL $=\{n 1, n 2, n 3, \ldots \ldots \ldots\}$

//Recommender nodes list

Step 5: Run the filtering algorithm

Step 6: Send the honest recommendation list to S \}

\subsection{Selection of honest recommenders}

In this section we have used multiple rules to select recommenders. These rules include majority opinion rule, personal knowledge rule and service reputation rules. These rules are used in combined to filter out the dishonest recommenders.

Majority Opinion Rule

In majority opinion rule, the trust approach takes majority opinion from all recommendations and categorizes them as per deviation test. The node whose deviation is very high from the majority opinion is treated as dishonest and ignores them from trust calculation.

\section{Personal Knowledge Rule}

The personal knowledge rule considers the node as malicious if its value deviates much from the opinion of the evaluating node. This rule applies deviation test to the receiving recommendations and eliminates those 
recommendations which have higher deviation value than the predefined threshold.

\section{Service Reputation Rule}

The service reputation rule consider that there is uniformity between service providing and recommendation. The recommendation from a reputed node is considered more trustworthy and given more weight while taken for evaluating trust. The recommendation can be taken on the basis of rank of the nodes. The nodes whose service reputation is more will be ranked as higher ranked nodes and will be given higher weightage in calculating recommendation.

Algorithm 2: Filtering Algorithm for honest recommendations

Step 1: For each recommendation list $L$ Do

Step 2: For each rating vector in the list $(\mathrm{x}, \mathrm{y})$ Do

Step 3: Calculate trust value for the recommender as equation (2)

Step 4: Calculate deviation values from all the recommenders $\quad d_{i k}=\left|D T_{i j}-R T_{k j}\right|$

Step 5: Rank the deviations as per $d_{i k}$, lowest $d_{i k}$ as highest rank and given highest

weightage.

Step 6: Weight $R T_{k j}$ as $W R T_{k j}=R T_{k j} * W_{k j}$ on the basis of $d_{i k}$ values

End For

Step 7: For each $d_{i k}$ Calculate $A v g d_{i k}$ End For

Step 8: If $\left(A v g d_{i k} \leq D\right)$ Then

// D is the deviation

threshold

Step 9: Return trustworthy Recommender End For

\section{EXPERIMENTAL SETUP}

The experiment is conducted in a MANET environment to test the performance of the proposed filtering algorithms for alleviating the impact of dishonest recommendations. In this experiment false rating data is propagated to simulate the ballot stuffing and bad mouthing problem. The aim of the experiment is the proper selection of recommenders for calculating the trustworthiness of a certain node in the presence of attacks. The simulation is performed in NS2 simulator with adding the required module to the simulator. We have used 60 random nodes in an area of $500 \mathrm{~m} \times 500 \mathrm{~m}$ area. For simulating bad mouthing and ballot stuffing attack several nodes are used to send false rating. Different scenarios are taken into considerations using different number of malicious nodes. We have taken a maximum of $50 \%$ misbehaving nodes. We have chosen the threshold value at 0.5 for considering the nodes as trusted. The Table 6.1 depicts the parameters used in the simulation process. Results are obtained after multiple run of the simulation.

\begin{tabular}{|l|l|}
\hline Parameter & Value \\
\hline No. of Nodes & 60 \\
\hline Area & $500 \mathrm{~m}$ X $500 \mathrm{~m}$ \\
\hline Speed & $30 \mathrm{~m} / \mathrm{s}$ \\
\hline Radio Range & $250 \mathrm{~m}$ \\
\hline Movement & $\begin{array}{l}\text { Random } \\
\text { waypoint model }\end{array}$ \\
\hline Routing Protocol & DSR \\
\hline MAC & 802.11 \\
\hline Application & CBR \\
\hline Packet size & $512 \mathrm{~B}$ \\
\hline Simulation time & $500 \mathrm{~s}$ \\
\hline Trust threshold & 0.5 \\
\hline Publication timer & $30 \mathrm{~s}$ \\
\hline $\begin{array}{l}\text { Deviation threshold, } \\
\text { D }\end{array}$ & 0.3 \\
\hline
\end{tabular}

\section{EXPERIMENTAL RESULTS}

There are several types of attacks which can occur due to dishonest recommendation problem. In this work only bad mouthing and ballot stuffing attacks are considered in proposed model. These two types of attacks are appropriate to show the performance of the model to minimize the dishonest recommendation problem. The expected trust value is computed in the simulation by considering the normal behavior of nodes. The model considers the average of the trust values recommended by other nodes. The simulation is carried out with the filtering algorithm and without the filtering algorithm with $0 \%$ to $50 \%$ of attacking nodes that is with no attacker node and half of the nodes is attacker. The simulation results are plotted in figure 1, 2 and 3.

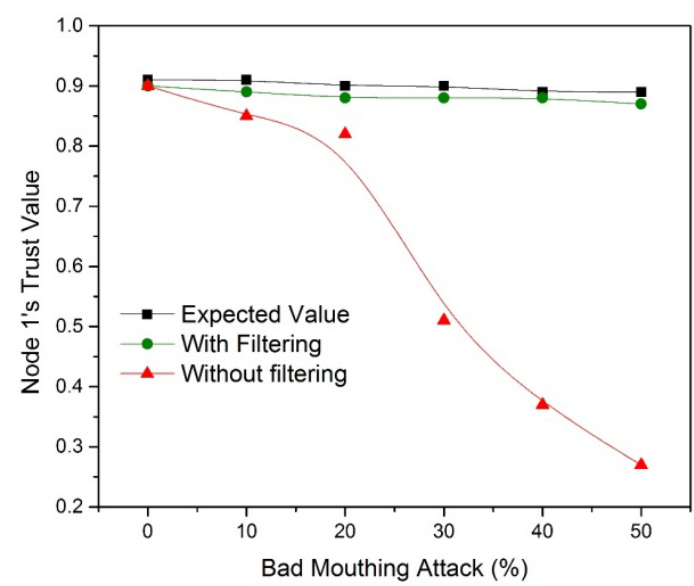

Figure 1: Trust value of node 1 in the presence of bad mouthing attack.

Figure 1 represents the trust value of a node which is considered as good; in this experiment node 1 is considered as good node. As the number of dishonest recommender
Table 1: Network Configuration Parameter used in Simulation 
nodes increases the trust value of node 1 is distorted when filtering algorithm is not applied because the dishonest recommenders propagate more false ratings. But from the figure it is clear that when filtering algorithm is applied, the trust value is as per expected value even when $50 \%$ nodes are considered as bad mouthing attackers.

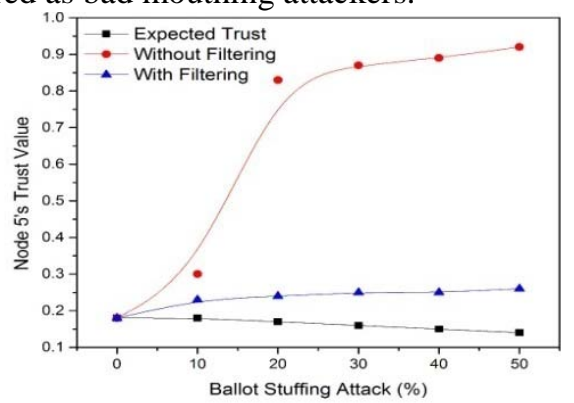

Figure 2: Trust value of node 5 in the presence of ballot stuffing attack

Figure 2 shows the effect of ballot stuffing attack. The figure shows the computed trust value of a bad node (node 5) when there are no dishonest recommender nodes, which evaluate the expected value; when there are dishonest recommendation nodes but filtering algorithm is disabled and when the filtering algorithm is active. The figure shows that the attacker nodes propagate dishonest recommendation for misleading the node while calculating the trust value. Thus when the attacker nodes percentage is $50 \%$, it can mislead the judgment by other nodes up to $90 \%$. The proposed filtering algorithm can mitigate the effect of dishonest recommendations to a reasonable extent. Figure 3 presents the influence of dishonest recommenders in the present of filtering algorithm and without the filtering algorithm on the basis of performance metrics throughput. Figure shows the throughput of simulated network with and without the defense scheme with the presence of $0 \%$ to $50 \%$ dishonest recommender nodes. The proposed defense scheme is able to maintain the throughput performance up to $80 \%$ even there is higher dishonest nodes. From the figure it is clear that dishonest recommender nodes can significantly effect on the network throughput. The proposed mechanism can maintain the throughput level up to a reasonable acceptable level even after there is a high increase in the dishonest nodes.

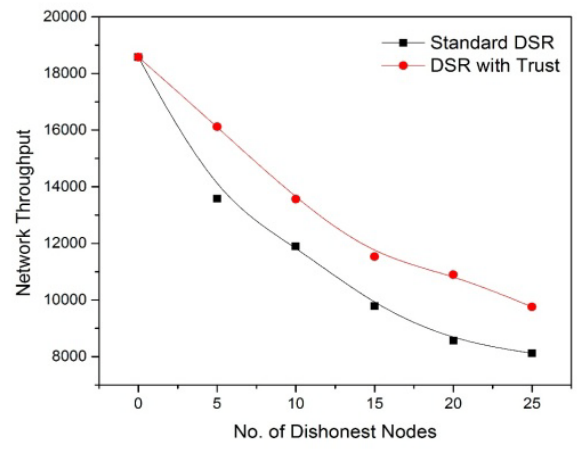

Figure 3: Throughput of the network
This paper presents a recommendation based trust model which uses probabilistic theory for calculating trust value. Here the ballot stuffing and bad mouthing attacks are considered while taking recommendations. This paper also addresses the dishonest recommender's problem which gives false recommendations about a node which leads to wrong trust value calculation. Here, a filtering algorithm is applied to resist the dishonest nodes from taking part in giving recommendations. A reasonable outcome is observed in resisting the ballot stuffing and bad mouthing attackers in producing dishonest recommendations. In future a more strict mechanism may be designed for proper authentication in taking recommendations so that the no attacker can attack the network.

\section{REFERENCES}

[1] Kannanand Mohapatra, (2012) Trust Computations and Trust Dynamics in Mobile Adhoc Networks: A Survey, IEEE 14(2), 279-298.

[2] R. Li, J. Li, P. Liu, and J. Kato, (2009), A Novel Hybrid Trust Management Framework for MANETs,in Distributed Computing Systems Workshops, 2009. ICDCS Workshops'09. 29th IEEE International Conference on,251-256.

[3] H. Yu, S. Liu, A. C. Kot, C. Miao, and C. Leung, (2011), Dynamic witness selection for trustworthy distributed cooperative sensing in cognitive radio networks, in Communication Technology (ICCT), 2011 IEEE 13th International Conference on, 1-

[4] C. Zouridaki, B. L. Mark, M. Hejmo, and R. K. Thomas, (2005) A quantitative trust establishment framework for reliable data packet delivery in MANETs, in Proceedings of the 3rd ACM workshop on Security of ad hoc and sensor networks,1-10.

[5] Buchegger, S. Le Boudec J.-Y., (2002), Performance analysis of the confident protocol (cooperation of nodes: fairness in dynamic ad-hoc networks) in MobiHoc'02, IEEE/ACM symposium on Mobile Ad-hoc Networking and Computing

[6] Tamilselvan, L. and Sankaranarayanan V., (2007), Prevention of Blackhole Attack in MANET, Proceedings of the $2^{\text {nd }}$ International Conference on Wireless Broadband and Ultra Wideband Communications, AUS

[7] Sun Choi, Doo-young Kim, Do-hyeon Lee, Jae-il Jung, (2008), WAP: Wormhole Attack Prevention Algorithm in Mobile Ad Hoc Networks, IEEE International Conference on Sensor Networks, Ubiquitous, and Trustworthy Computing

[8] N. Bhalaji, A. Shanmugam, (2012), Dynamic Trust Based Method to Mitigate Grayhole Attack in MobileAd-hoc Networks, International Conference on Communication Technology and System Design, Procedia Engineering 30, $881-888$

[9] K. Hoffman, D. Zage, and C. Nita-Rotaru, (2009) A survey of attack and defense techniques for reputation systems, ACM Computing Surveys (CSUR),1-9.

\section{SUMMARY AND CONCLUSION}

